\title{
Patterning of graphene on silicon-on-insulator waveguides through laser ablation and plasma etching
}

\author{
Jürgen Van Erps ${ }^{a *}$, Tymoteusz Ciuk $^{b}$, Iwona Pasternak ${ }^{b}$, Aleksandra Krajewska ${ }^{b}$, \\ Wlodek Strupinski ${ }^{b}$, Steven Van Put $^{c}$, Geert Van Steenberge ${ }^{c}$, Kitty Baert ${ }^{d}$, Herman Terryn ${ }^{d}$, \\ Hugo Thienpont ${ }^{a}$, and Nathalie Vermeulen ${ }^{a}$ \\ ${ }^{a}$ Vrije Universiteit Brussel, Brussels Photonics Team (B-PHOT), \\ Department of Applied Physics and Photonics, Pleinlaan 2, B-1050 Brussel, Belgium \\ ${ }^{b}$ Institute of Electronic Materials Technology, Wolczynska 133, 01-919 Warsaw, Poland \\ ${ }^{c}$ Centre for Microsystems Technology (CMST), imec and Ghent University, \\ Technologiepark 914A, B-9052 Gent, Belgium \\ ${ }^{d}$ Vrije Universiteit Brussel, Research Group Electrochemical and Surface Engineering (SURF), \\ Pleinlaan 2, B-1050, Belgium
}

\begin{abstract}
We present the use of femtosecond laser ablation for the removal of monolayer graphene from silicon-on-insulator (SOI) waveguides, and the use of oxygen plasma etching through a metal mask to peel off graphene from the grating couplers attached to the waveguides. Through Raman spectroscopy and atomic force microscopy, we show that the removal of graphene is successful with minimal damage to the underlying SOI waveguides. Finally, we employ both removal techniques to measure the contribution of graphene to the loss of grating-coupled graphenecovered SOI waveguides using the cut-back method. This loss contribution is measured to be $0.132 \mathrm{~dB} / \mu \mathrm{m}$.
\end{abstract}

Keywords: graphene, laser ablation, loss measurement, plasma etching, Raman spectroscopy, silicon photonics, waveguides

\section{INTRODUCTION}

In recent years graphene, a two-dimensional hexagonal lattice of carbon atoms, has been shown to exhibit unique optical properties that can strongly improve the functioning of photonic devices and even of entire photonic circuits. ${ }^{1,2}$ When depositing graphene on chip-scale photonic circuits consisting of silicon waveguides, a high-resolution patterning method to locally remove the graphene layer without affecting the underlying silicon waveguide is desired for most applications. High-resolution patterning of graphene on silicon waveguides is typically established using photolitography or electron beam lithography. ${ }^{3,4}$ Yet there are important limitations associated with these techniques, such as minimal wafer size requirements which prohibit chip-scale processing. Most importantly, however, the subsequent steps of lithography may seriously damage the underlying graphene sheet or at least significantly alter its electrical and optical properties through chemicals-induced unintentional doping.

A promising alternative technique for realizing high-resolution graphene patterning without emplying chemicals is laser ablation. ${ }^{5-7}$ Its viability has already been demonstrated for graphene deposited on $\mathrm{SiO}_{2}$, but ablation of graphene directly deposited on silicon has not yet been reported. The challenge here is to properly select the laser parameters since silicon, as opposed to $\mathrm{SiO}_{2}$, absorbs infrared light with wavelengths shorter than $1100 \mathrm{~nm}$ and thus is more prone to laser-induced damage at these wavelengths. Furthermore, laser ablation of graphene on waveguide structures - regardless the material used - has to our knowledge never been investigated.

In this paper we show that laser ablation can successfully remove graphene on a silicon waveguide without deteriorating the waveguide shape. We also find that laser ablation is not suitable for peeling off graphene from

*E-mail: Jurgen.Van.Erps@vub.ac.be, Tel.: +32 4978007 94, Fax.: +32 262934 50, http://www.b-phot.org. B-PHOT is a member of Flanders Make.

Silicon Photonics and Photonic Integrated Circuits V, edited by Laurent Vivien, Lorenzo Pavesi, Stefano Pelli, Proc. of SPIE Vol. 9891, 98910T - (c) 2016 SPIE · CCC code: 0277-786X/16/\$18 · doi: 10.1117/12.2225224 


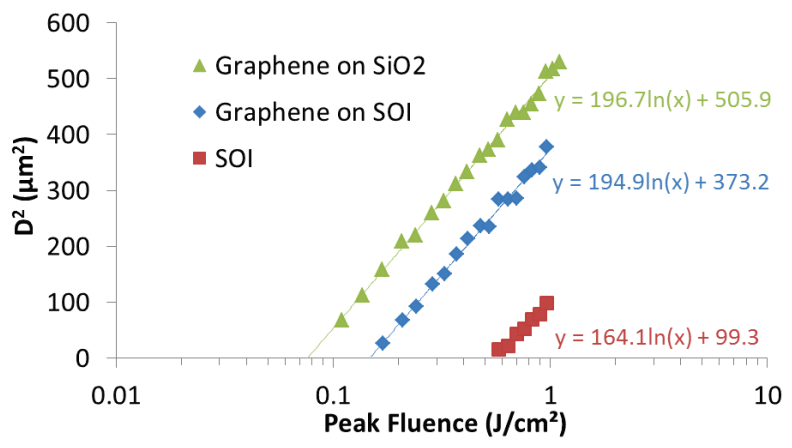

Figure 1. Experimental measurement of the squared diameter of the ablated areas $\left(\mathrm{D}^{2}\right)$ as a function of the peak fluence to determine the ablation threshold, which is the intersection of the fitted curve with the X-axis.

the grating couplers attached to the waveguide input and output to enable light incoupling and outcoupling. Instead, oxygen plasma etching through a mechanical mask can be employed for this purpose. As a case study, we apply both graphene removal techniques to determine the linear loss of grating-coupled graphene-covered silicon waveguides along the cut-back method. ${ }^{8}$

\section{MONOLAYER GRAPHENE TRANSFER}

Monolayer graphene was grown on high-purity polycrystalline copper foil in a commercial Black Magic system by the chemical vapor deposition (CVD) method. The pre-growth treatment of copper included annealing at $1000^{\circ} \mathrm{C}$ in $\mathrm{Ar}$ atmosphere followed by $\mathrm{H}_{2}$ etching at a pressure of 20 mbar. ${ }^{9}$ The purpose of this step is to improve the quality and enlarge the grain size of copper as well as to ensure a reduction of oxides from the copper surface. Later, methane was introduced into the reactor with time and flow rate settings ensuring monolayer graphene growth. The as-grown graphene was covered with a $200 \mathrm{~nm}$-thin layer of PMMA by spin-coating and transferred onto an SOI photonic chip by means of the electrochemical delamination (ED) method carried out in $1 \mathrm{M}$ aqueous solution of potassium chloride. ${ }^{9}$

\section{FEMTOSECOND LASER ABLATION}

Graphene ablation was investigated using an Yb-doped fiber laser (Satsuma, Amplitude Systems, $5 \mathrm{~W}, 1030 \mathrm{~nm}$, $<400 \mathrm{fs}$ ). During the ablation experiments, the pulse repetition rate was fixed to $10 \mathrm{kHz}$, whereas the laser pulse energy and the amount of shots (i.e. the laser scanning speed) were varied. An objective lens with a focal length of $60 \mathrm{~mm}$ was used to focus the laser beam and all ablation experiments were carried out in air at room temperature. The spot size of the laser beam was determined ${ }^{10}$ to be $w_{0}=9.9 \mu \mathrm{m}$.

We started with investigating laser ablation of a monolayer graphene sheet (with the PMMA layer removed) on an $\mathrm{SiO}_{2}$ substrate. The graph in Figure 1 shows the squared diameter of the ablated areas $\left(\mathrm{D}^{2}\right)$ as a function of the peak fluence for single shots. From this graph, the ablation threshold was determined to be $0.076 \mathrm{~J} / \mathrm{cm}^{2}$. This ablation threshold is higher than the value found by Zhang et al., ${ }^{11}$ but they worked at a different wavelength, namely $800 \mathrm{~nm}$.

To selectively remove monolayer graphene deposited on an SOI substrate with a $220 \mathrm{~nm}$-thick top silicon layer, we found that the ablation threshold for graphene removal, i.e. $0.147 \mathrm{~J} / \mathrm{cm}^{2}$ (Figure 1), was a factor 2 higher for graphene on silicon than for graphene on $\mathrm{SiO}_{2}\left(0.076 \mathrm{~J} / \mathrm{cm}^{2}\right)$, while the damage threshold for silicon was $0.546 \mathrm{~J} / \mathrm{cm}^{2}$ (Figure 1). Having determined the threshold for ablation of graphene on a blank SOI sample, we proceeded to the local removal of graphene on a SOI photonic chip featuring a $220 \mathrm{~nm}$-thick top silicon layer. The chip contained $450 \mathrm{~nm}$-wide waveguides fabricated in the $220 \mathrm{~nm}$-thick silicon top layer, which are the standard waveguide dimensions for operation at the telecom wavelength of $1550 \mathrm{~nm}$. The waveguides' grating couplers were also designed for $1550 \mathrm{~nm}$. We found a fluence of $0.16 \mathrm{~J} / \mathrm{cm}^{2}$ (corresponding to an average power of $2.5 \mathrm{~mW}$ ), just above the ablation threshold, and a laser scanning speed of $70 \mathrm{~mm} / \mathrm{s}$ to be the optimal parameters for continuous removal of graphene from the silicon waveguides without damaging the waveguides themselves. To show that the local removal of graphene was successful, we measured the Raman spectrum at a position 


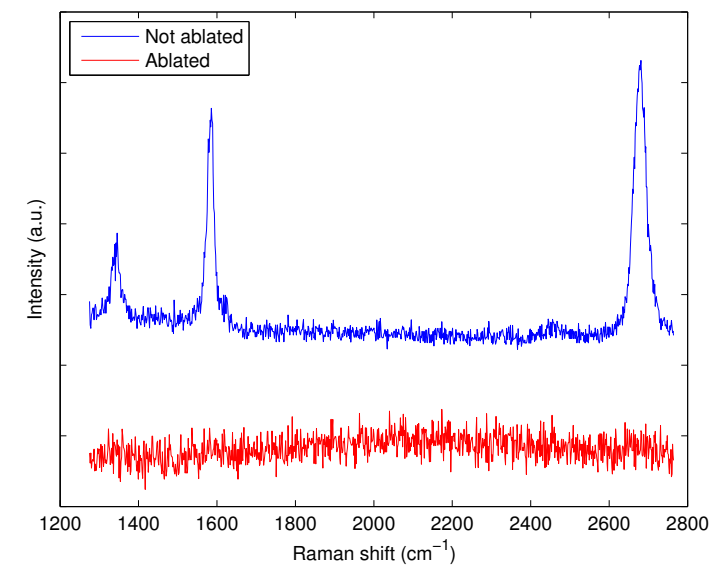

Figure 2. Raman spectra measured in the ablated and non-ablated regions of a graphene-covered SOI waveguide.
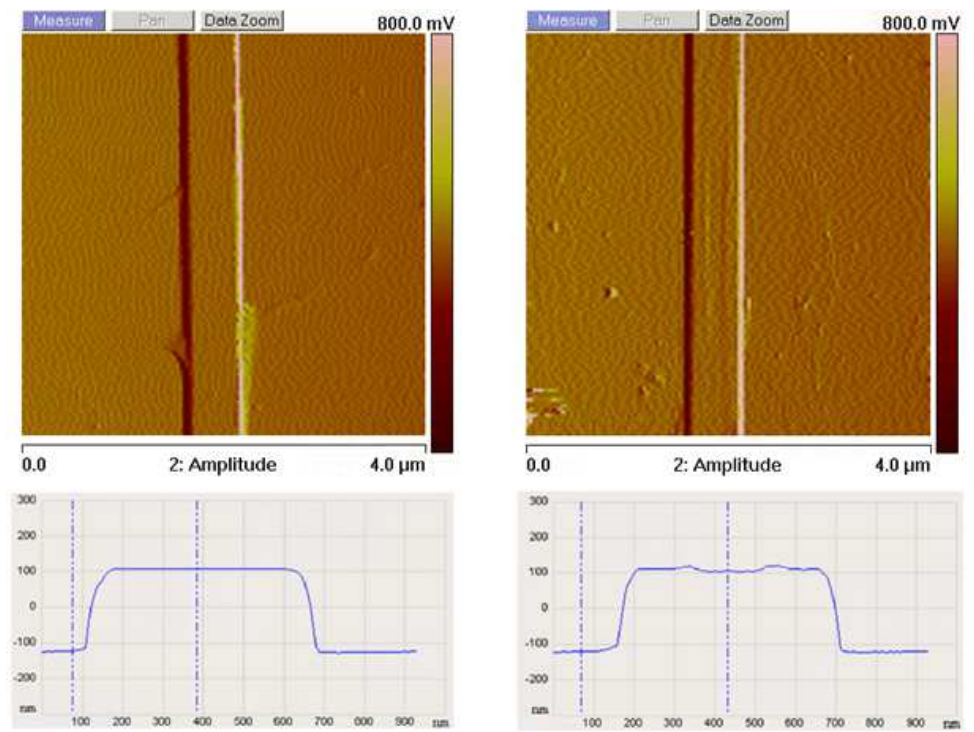

Figure 3. AFM measurements showing the waveguide profile before ablation (left) and after ablation (right). The SOI waveguides have a height of $220 \mathrm{~nm}$ and a width of $450 \mathrm{~nm}$.

on the waveguide that was ablated and at a position that was not ablated. Figure 2 shows a typical result of these measurements. Whereas in the blue curve the characteristic peaks clearly show the presence of monolayer graphene, the red curve shows that there is no graphene remaining on top of the waveguide after laser ablation. In addition, atomic force microscopy (AFM) was used to check the geometrical shape and surface roughness of the SOI waveguides after laser ablation, to identify potential damage to the waveguide during ablation. A comparison of the surface profile of an SOI waveguide before and after ablation is shown in Figure 3. A slight ablation-induced increase in surface roughness can be observed in the right-hand side. From these measurements, we found that the parameter window for graphene removal with minimal damage to the underlying waveguide is relatively small.

We also observed that whereas for the waveguides the selective removal of graphene with laser ablation was successful, it was not possible to achieve the same result on the grating couplers. We believe that resonant reflections of the laser ablation beam on the gratings results in damage to the gratings even at very low ablation powers. For the tapered SOI sections between the waveguides and the grating couplers, we found that the level of adhesion of the graphene layer depends on the local width of the tapers, so that removing the graphene along the tapers would require a gradual variation of the laser ablation settings along the taper. To avoid this and to solve the issue of graphene removal from the grating couplers, we used $\mathrm{O}_{2}$ plasma etching ${ }^{12,13}$ to peel off the 


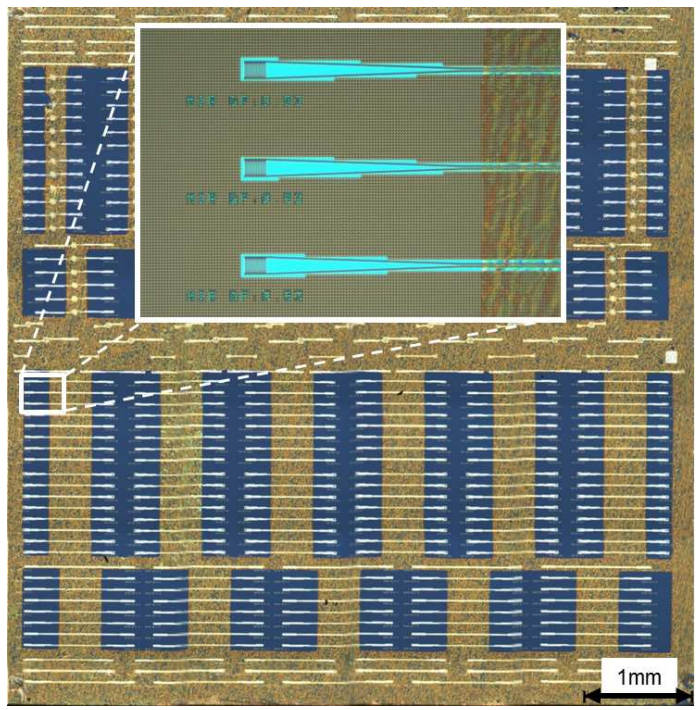

Figure 4. Microscope image of the photonic chip on which a PMMA/graphene layer has been transferred, which is then selectively etched to create openings in the layer on top of the grating couplers and tapered sections of the SOI waveguides. Inset: zoom on three grating couplers in an etched opening, and the transition to a graphene-covered region (i.e. the slightly darker stripe that runs vertically across the figure).

graphene from the grating couplers and the tapers.

\section{OXYGEN PLASMA ETCHING}

As opposed to laser ablation, the plasma etching step can be performed before the PMMA layer is washed off of the graphene sheet. The $\mathrm{O}_{2}$ plasma etching was carried out in a $13.56 \mathrm{MHz}$ chemically reactive plasma etcher. The oxygen flow was set to $5 \mathrm{sccm}$ and the pressure reached 0.3 mbar. At $50 \mathrm{~W}$ of applied RF power the etching continued for $30 \mathrm{~min}$. A mechanical mask was designed and laser-cut in a $200 \mu \mathrm{m}$-thin steel plate to define the openings to be etched. The alignment of the mask with respect to the chip proved a challenging task and was carried out with the help of custom-adapted equipment which allowed us to achieve an alignment accuracy of $10 \mu \mathrm{m}$. Figure 4 shows the SOI chip after the mask alignment has been carried out and openings have been etched in the graphene/PMMA layer. The brown area indicates where the graphene/PMMA layer was still present and the dark blue area where it was etched away. The selectively etched graphene/PMMA layer is afterwards rinsed in an acetone/ethanol bath to strip the PMMA layer and reveal the underlying graphene. The inset of Figure 4 shows a zoom of three grating couplers situated within one of the etched openings. Figure 5 shows the Raman spectrum of graphene outside the $\mathrm{O}_{2}$ plasma etching region, showing that the remaining graphene monolayer is of good quality. In the same figure, the Raman spectrum taken within the etched area exhibits no graphene-related peaks and shows no traces of carbon residue. This clearly shows that the $\mathrm{O}_{2}$ plasma completely removes the graphene layer in the selectively etched areas.

\section{MEASUREMENT OF LINEAR LOSS OF GRAPHENE-COVERED WAVEGUIDES USING CUT-BACK METHOD}

To showcase its practical use for optical experiments, we also employed our combined laser ablation and plasma etching technique for determining the optical transmission properties of graphene-covered silicon waveguides. More specifically, we have plasma etched and laser ablated the gratings and waveguides according to the pattern shown in Figure 6. This way, the length of the graphene-covered waveguide is gradually increased from $0 \mu \mathrm{m}$ (i.e. the graphene is removed over the full waveguide length) to $300 \mu m$ such that we can determine the linear propagation losses by means of the so-called cut-back method. ${ }^{14}$

To couple light between optical single-mode fibers and the silicon waveguides, we positioned the fibers above the grating couplers at an angle of $9^{\circ}$ with respect to the vertical axis for optimal coupling efficiency. As a 


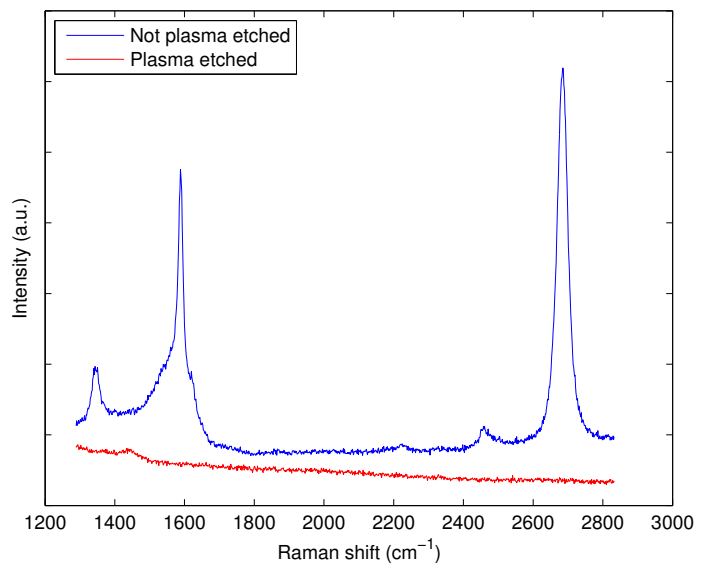

Figure 5. Raman spectra measured in a graphene-covered region and in the oxygen plasma etched grating coupler area.

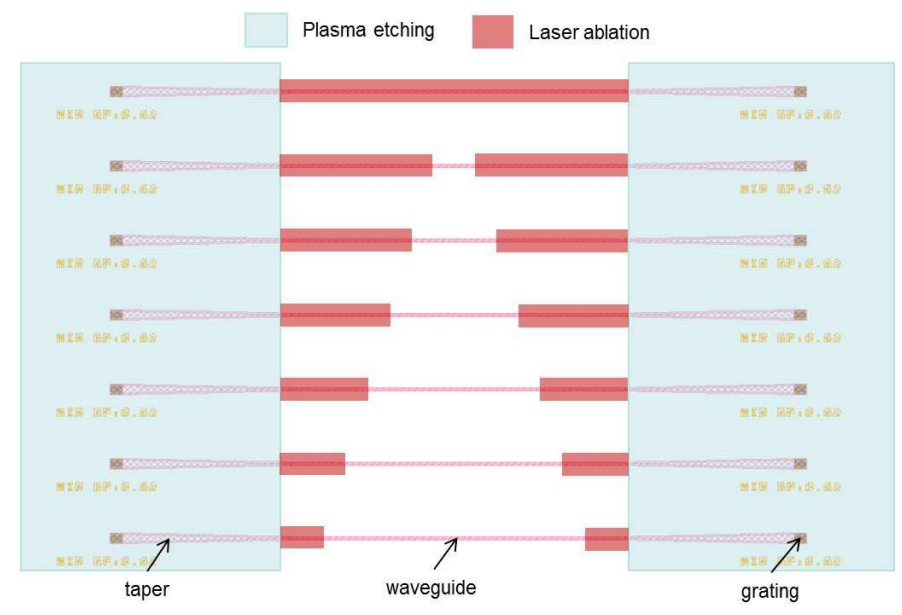

Figure 6. Schematic lay-out of the laser ablation and $\mathrm{O}_{2}$ plasma etching regions on an array of identical graphene-covered waveguides, to be used for cut-back measurements.

reference, we first measured the grating coupler efficiency before graphene transfer on the chip. The source employed is a continuous-wave wavelength-division multiplexing telecom laser (emitting at the wavelength of $1550 \mathrm{~nm}$ for which the grating couplers were designed) connected to an Erbium-doped fiber amplifier set to $1 \mathrm{~mW}$ output power. The total insertion loss, including the grating coupler loss at input and output and the waveguide propagation loss over $800 \mu \mathrm{m}$ (the latter comprises a $400 \mathrm{um}$-long waveguide section and two $200 \mathrm{um}-$ long tapered sections) was found to be $13.5 \mathrm{~dB}$. Since the propagation loss of the waveguides is of the order of $3 \mathrm{~dB} / \mathrm{cm},{ }^{14}$ the insertion loss per grating is estimated to be $6.6 \mathrm{~dB}$ when using flat-cleaved fibers to couple light in and out of the SOI waveguides.

Then we performed the cut-back characterization of the graphene-covered waveguides. Figure 7 shows the total insertion loss (i.e. the waveguide loss and the coupling loss at both grating couplers) versus the length of the graphene strip in-between the ablated zones. The leftmost data point in Figure 7 shows a total insertion loss of $18.3 \mathrm{~dB}$ for a waveguide which was ablated over its full length. This is about $4.8 \mathrm{~dB}$ higher than the insertion loss of $13.5 \mathrm{~dB}$ measured before the graphene transfer and ablation. This difference is likely due to the ablation-induced increase in surface roughness of the silicon waveguide, as mentioned before and which can be observed in Figure 3.

When applying a linear regression on all datapoints in Figure 7, we find that the contribution of graphene to the propagation loss for the graphene-covered waveguides is about $13.2 \mathrm{~dB}$ per $100 \mu \mathrm{m}$ of graphene, with a very good $\mathrm{R}^{2}$ value of 0.9925 for the fit. This loss of $0.132 \mathrm{~dB} / \mu \mathrm{m}$ is in line with other experimental results ${ }^{15}$ and is strongly dependent on the waveguide geometry. 


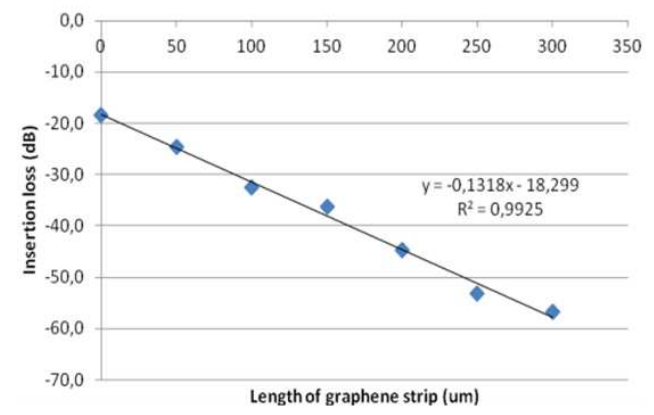

Figure 7. Results of the linear transmission cut-back experiments.

\section{CONCLUSIONS}

In conclusion, we have demonstrated the successful selective removal of a monolayer of graphene on SOI waveguides through femtosecond laser ablation at $1030 \mathrm{~nm}$. Because laser ablation of the grating couplers was not successful, we employed $\mathrm{O}_{2}$ plasma etching through a metal mask to remove graphene at these locations, as well as on the tapered waveguide sections. Atomic force microscopy revealed that laser ablation induced a slight increase in surface roughness of the underlying waveguides, which resulted in a $4.8 \mathrm{~dB}$ insertion loss penalty for a 400 um-long ablated waveguide section. Both the laser ablation and plasma etching techniques offer the important advantage that they do not involve chemical treatment of the graphene sheet. Finally, we employed both techniques for determining the optical transmission of graphene-covered SOI waveguides along the cut-back method. For a standard waveguide width of $450 \mathrm{~nm}$ and height of $220 \mathrm{~nm}$ we found the contribution of graphene to the optical loss to be $0.132 \mathrm{~dB} / \mu \mathrm{m}$. This high loss value per unit distance clearly shows that to apply graphene in photonic chip applications, accurate control of the overall loss through localized patterning of the graphene is essential.

\section{ACKNOWLEDGMENTS}

This work was supported by the EU-FET GRAPHENICS (grant agreement no. 618086), BELSPO-IAP, the Methusalem foundation, ERC-FP7/2007-2013 grant 336940 and by the EU-FP7 Graphene Flagship (grant agreement no. 604391).

\section{REFERENCES}

[1] Cheng, J.L., Vermeulen, N., Sipe, J.E., "Third Order Optical Nonlinearity of Graphene," New J. Phys. 16, 053014 (2014).

[2] Cheng, J.L., Vermeulen, N., Sipe, J.E., "DC Current Induced Second Order Optical Nonlinearity in Graphene," Opt. Express 22, 15868-15876 (2014).

[3] Ye, L., Gan, L., Dai, L., Dai, Y., Guo, X., Meng, H., Yu, B., Shi, Z., Shang, K., Qin, G., "A Simple and Scalable Graphene Patterning Method and Its Application in CdSe Nanobelt/Graphene Schottky Junction Solar Cells," Nanoscale 11, 1477-1481 (2011).

[4] Kim, K.S., Zhao, Y., Jang, H., Lee, S.Y., Kim, J.M., Kim, K.S., Ahn, J.-H., Kim, P., Choi, J.-Y., Hong, B.H., "Large-Scale Pattern Growth of Graphene Films for Stretchable Transparent Electrodes," Nature 457, 706-710 (2009).

[5] Sahin, R., Simsek, E., Akturk, S., "Nanoscale Patterning of Graphene through Femtosecond Laser Ablation," Appl. Phys. Lett. 104, 053118 (2014).

[6] Wakaya, F., Kurihara, T., Abo, S., Takai, M., "Ultra-violet laser processing of graphene on $\mathrm{SiO}_{2} / \mathrm{Si}$," $M i$ croelectron. Eng. 110 358-360 (2013).

[7] Bobrinetskiy, I., Emelianov, A.V., Otero, N., Romero, P.M., "Patterned Graphene Ablation and Two-Photon Functionalization by Picosecond Laser Pulses in Ambient Conditions," Appl. Phys. Lett. 107, 043104 (2015).

[8] Van Erps, J., Ciuk, T., Pasternak, I., Krajewska, A., Strupinski, W., Van Put, S., Van Steenberge, G., Baert, K., Terryn, H., Thienpont, H., Vermeulen, N., "Laser ablation- and plasma etching-based patterning of graphene on silicon-on-insulator waveguides," Opt. Express 23, 26639-26650 (2015). 
[9] Ciuk, T., Pasternak, I., Krajewska, A., Sobieski, J., Caban, P., Szmidt, J., Strupinski, W., "Properties of Chemical Vapor Deposition Graphene Transferred by High-Speed Electrochemical Delamination," J. Phys. Chem. C 117, 20833-20837 (2013).

[10] Liu, J.M., "Simple Technique for Measurements of Pulsed Gaussian-beam Spot Sizes," Opt. Lett. 7, 196-198 (1982).

[11] Zhang, W., Li, L., Wang, Z.B., Pena, A.A., Whitehead, D.J., Zhong, M.L., Lin, Z., Zhu, H.W., "Ti:Sapphire Femtosecond Laser Direct Micro-Cutting and Profiling of Graphene," Appl. Phys. A 109, 291-297 (2012).

[12] Al-Mumen, H., Rao, F., Li, W., Dong, L., "Singular Sheet Etching of Graphene with Oxygen Plasma," Nano-Micro Lett. 6, 116-124 (2014).

[13] Childres, I., Jauregui, L.A., Tian, J., Chen, Y.P., "Effect of Oxygen Plasma Etching on Graphene studied using Raman Spectroscopy and Electronic Transport Measurements," New J. Phys. 13, 025008 (2011).

[14] Vlasov, Y.A., McNab, S.J., "Losses in Single-Mode Silicon-on-Insulator Strip Waveguides and Bends," Opt. Express 12, 1622-1631 (2014).

[15] Li, H., Anugrah, Y., Koester, S.J., Li, M., "Optical Absorption in Graphene Integrated on Silicon Waveguides," Appl. Phys. Lett. 101, 111110 (2012). 